\title{
Adam, sag, wie hast du's mit der Religion?* \\ Religion und Kirche im Werk Adam Smiths
}

\section{MiCHAEL S. AßLÄNDER ${ }^{* *}$}

Die religiöse Einstellung Adam Smiths bildet seit mehr als 200 Jahren den Gegenstand zahlreicher Diskussionen und Interpretationsversuche. Bis heute ist der Stellenwert, den Religion in Smiths Leben und Werk einnimmt, weitgehend ungeklärt. These des folgenden Beitrages ist, dass Smith eine deistische Weltsicht vertritt, die als verbindende Klammer zwischen seinen Hauptwerken, Theory of Moral Sentiments und Wealth of Nations, interpretiert werden kann. Wie gezeigt werden soll, beruht Smiths Vorstellung einer harmonischen Weltordnung dabei wesentlich auf den Ideen seines akademischen Lehrers Francis Hutcheson.

Schlagwörter: Adam Smith, Francis Hutcheson, Religion, Deismus, Toleranz, Institutionen, Erziehung

\section{Adam, tell me, where do you stand in terms of religion? - Religion and Church in the Writings of Adam Smith}

For more than 200 years, the religious beliefs of Adam Smith have been subject of academic discussions and interpretations. Till nowadays, the importance of religion in Smith's life and in bis work remains unclear. As I will argue in the following, Smith follows a deistic worldview which might be seen as the common bond linking his Theory of Moral Sentiments to bis Wealth of Nations. As I will show, Smith's concept of a harmonic world-order is based largely on the ideas of his academic teacher Francis Hutcheson.

Keywords: Adam Smith, Francis Hutcheson, Religion, Deism, Tolerance, Institutions, Education

\section{Selbstliebe statt Sympathie?}

Die religiöse Einstellung Adam Smiths bildet seit mehr als 200 Jahren den Gegenstand zahlreicher Diskussionen und Interpretationsversuche (vgl. u.a. Rothschild 2002; Raphael 2007; Luterbach-Maineri 2008). Dabei ist der Stellenwert, den Religion in Smiths Leben und Werk einnimmt, weitgehend ungeklärt und wird bis heute kontrovers diskutiert. Für Smiths persönliche religiöse Einstellung, so Knud Haakonssen (vgl. 2006: 13f.), fehlt es an Belegen und auch die Einschätzung des Stellenwerts der Religion in seinem Werk bleibt schwierig. Umstritten ist, ob Smiths Theorieentwurf

\footnotetext{
* Beitrag eingereicht am 07.03.2016; nach doppelt verdecktem Gutachterverfahren überarbeitete Fassung angenommen am 18.09.2016.

** Prof. Dr. Michael S. Aßländer, Technische Universität Dresden, Internationales Hochschulinstitut Zittau, Markt 23, D-02763 Zittau, E-Mail: michael.asslaender@gmx.de, Forschungsschwerpunkte: Angewandte Ethik, Wirtschaftsethik, Arbeitsbegriff, Theoriegeschichte der Ökonomie.
} 
wesentlich auf religiösen, i.e. deistischen Moralvorstellungen aufbaut oder ob die religiöse Metaphorik lediglich dem „Zeitgeist“ einer zutiefst religiös geprägten Gesellschaft an der Schwelle zur Aufklärung geschuldet ist. So sieht es etwa Josef Schumpeter als Charakteristikum der englischen Nationalökonomen, dass diese „mit dem soliden Rüstzeug anglikanischer Theologie auf ihre intellektuellen Reisen gingen“ (Schumpeter 2009: 944 FN25), was cum grano salis auch für das Verhältnis der Schottischen Aufklärung zur presbyterianischen Kirche gelten mag.

Geführt wird der Streit um Smiths religiöse Einstellung insbesondere vor dem Hintergrund zweier zentraler Annahmen in seinen theoretischen Entwürfen: dem impartial spectator als unparteiischen Richter über die Angemessenheit des Verhaltens grundsätzlich sympatbiefähiger Akteure, den Smith in seiner Theory of Moral Sentiments (TMS) einführt, und der invisible hand als Ausdruck einer prästabilierten Harmonie, die das Verhalten primär eigennutzorientierter Akteure zum Wohle der Allgemeinheit wirksam werden lässt, und die nach Meinung zahlreicher Interpreten insbesondere in seinem ökonomischen Hauptwerk, der Inquiry into the Nature and Causes of the Wealth of Nations (WN), zur moralischen Rechtfertigung rein ökonomischer Austauschbeziehungen diene. Damit scheinen zwei unterschiedliche Ordnungsinstanzen einander gegenüberzutreten. Während in Fragen der Moral der unparteiische Zuschauer, als „Statthalter [...], den die Gottheit in uns eingesetzt hat" (Smith 1985a [1759]: 250), über die moralische Angemessenheit unserer Absichten, unseres Handelns und unsere Affekte urteilt (vgl. Hanley 2009: 141f.), scheint der Mensch in ökonomischen Dingen frei von Moral seinen eigenen ökonomischen Vorteil erstreben zu können, im Vertrauen darauf, dass er durch sein eigennütziges Handeln „ohne es zu beabsichtigen, ja ohne es zu wissen, das Interesse der Gesellschaft“ (Smith 1985a [1759]: 316f.) fördert; dabei dient „er in der Regel nicht bewusst [dem] Allgemeinwohl, noch weiß er, wie hoch der eigene Beitrag ist“" aber „er wird in diesem wie auch in vielen anderen Fällen von einer unsichtbaren Hand geleitet, um einen Zweck zu fördern, den zu erfüllen er in keiner Weise beabsichtigt hat" (Smith 1990 [1776]: 371).

Insbesondere im deutschsprachigen Raum führte dies zu der als Umschwnngtheorie ${ }^{1}$ bekannt gewordenen Annahme, Smith habe sich im Laufe seines akademischen Schaffens vom Idealisten zum Materialisten entwickelt und setze in seinem späteren Werk den rein am materiellen Wohlergehen orientierten Egoisten anstelle des empathiefähigen und tugendhaften Menschen seiner TMS ein. Bis heute wird dieses Adam-SmithProblem $^{2}$ zweier entgegengesetzter Menschenbilder innerhalb von Moralphilosophie

1 Während Lujo Brentano Smiths „Umschwung [...] in seinen Grundanschauungen“ vor allem auf den Einfluss von Claude Adrien Helvétius' Schrift De l'esprit zurückführen zu können glaubt (vgl. Brentano 1877: 60f.), in der die „Selbstliebe“ eine zentrale Stelle einnimmt, geht Witold von Skarzynski davon aus, dass die Änderung in den Ansichten Smiths generell dem Einfluss des französischen Materialismus geschuldet sei: „Unter dem Einfluss Hutchesons und Humes war Smith Idealist, so lange er in England blieb. Nach dreijähriger Berührung mit dem Materialismus, der in Frankreich herrschte, kehrte er nach England als Materialist zurück“ (Skarzynski 1878: 183).

2 Vgl. zur frühen Diskussion u.a. Wilhelm Paszkowski (1890) und August Oncken (1898); zur aktuellen Debatte vgl. u.a. Nutzinger (1991: 85-92), Werhane (2000) u. Paganelli (2008). Ausführlich hierzu auch: Patzen (1991), Eckstein (1985: LIII-LXVI), Raphael/Macfie (1982: 2025) und Aßländer (2007: 147-158). 
und Ökonomie kontrovers diskutiert. Ohne im Folgenden näher auf die Geschichte dieses Streites eingehen zu wollen, kann festgehalten werden, dass beide Menschenbilder durchaus als miteinander in Einklang stehend betrachtet werden können. Für Smith ergibt sich Selbstliebe nicht aus dem Egoismus böswilliger Individualisten, sondern aus dem Tugendgebot der Klugheit; während hingegen die Wohltätigkeit gegenüber unseren Mitmenschen den Tugendgeboten der Gerechtigkeit und des Wohlwollens folgt (vgl. Oncken 1898: 105; Merikoski 2002: 61ff.). All diese Tugenden sind dem Menschen von Natur aus gleichermaßen gegeben, um die menschliche Glückseligkeit zu befördern (vgl. Luterbacher-Maineri 2008: 143); sie sind Teil der prästabilierten harmonischen Ordnung des Kosmos. Denn so „oft wir durch natürliche Triebe dazu bestimmt werden, jene Zwecke zu fördern, die eine verfeinerte und aufgeklärte Vernunft uns anempfehlen würde, dann sind wir geneigt, [...] uns einzubilden, dasjenige sei die Weisheit von Menschen, was in Wirklichkeit die Weisheit Gottes ist" (Smith 1985a [1759]: 130).

Betrachtet man Smiths Schriften also auf Folie der Annahme einer von Smith stets unterstellten, weise eingerichteten und harmonischen Weltordnung, in der alle Teile auf wunderbare Weise zusammenwirken, ${ }^{3}$ um den Zweck der Schöpfung zu verwirklichen, löst sich der scheinbare Widerspruch des Adam-Smith-Problems auf: Selbstliebe, Wohlwollen und Sinn für Gerechtigkeit wirken ebenso zum Wohle des Ganzen, wie das Urteil des unparteiischen Zuschauers und der Mechanismus der unsichtbaren Hand. So gesehen bildet das deistische Verständnis einer harmonischen Weltordnung die verbindende Klammer zwischen Smiths beiden Hauptwerken. Obwohl Smith kurz vor seinem Tode wesentliche Passagen der TMS verändert und nun eine stärkere Distanz zum christlichen Offenbarungsglauben zeigt, bleibt er prinzipiell auch in der sechsten Auflage der TMS dieser Weltsicht treu. In gewisser Weise dienen die Veränderungen sogar der Präzisierung seiner Ideen und können, wie Maria Paganelli (vgl. 2011: 131) es ausdrückt, als ein „update“ der bisherigen Gedanken gelesen werden.

These des folgenden Beitrages ist, dass Smith in seiner deistischen Weltsicht wesentlich von der Moralphilosophie Francis Hutchesons geprägt ist. Insbesondere die TMS folgt dabei grundsätzlich der Sicht Hutchesons und basiert auf der Annahme sympathiefähiger und am Wohlergehen ihrer Mitmenschen interessierter Akteure. Doch auch die oftmals als Grundlage eines Laissez-faire-Liberalismus missverstandene Annahme der primär an ihrem eigenen materiellen Wohlergehen interessierten Wirtschaftsakteure im WN folgt Hutchesons Annahme eines moderaten Egoismus, der den einzelnen dazu bestimmt, aufgrund von Klugheitsüberlegungen seine eigenen Interessen zu wahren. Um diese These zu untermauern, soll im Folgenden zunächst auf die Grundzüge der Hutchesonschen Moralphilosophie näher eingegangen werden. In einem zweiten Schritt gilt es, das hierauf aufbauende harmonische Weltverständnis Smiths als Grundlage sowohl seiner Moralphilosophie als auch seiner Wirtschaftstheo-

Smith selbst vergleicht diese Wirkungsweise mit den Rädern einer Uhr, die vom Uhrmacher geschaffen sind, um ihren Zweck, die Zeitmessung, zu erfüllen und deren „Bewegungen [...] in der genauesten Weise“ zusammenwirken und die es nicht vollkommener tun könnten, „wenn sie mit dem Wunsch oder der Absicht, diesen Zweck zu erreichen, begabt wären. Doch schreiben wir einen solchen Wunsch oder eine solche Absicht niemals ihnen zu, sondern dem Uhrmacher..." (Smith 1985a [1759]: 130). Vgl. hierzu auch Büscher (1991: 126ff.). 
rie näher zu durchleuchten. In einem weiteren Schritt soll auf Smiths Idee eines Markts der Religionen eingegangen werden, eine der wenigen Stellen, an denen Smith sich explizit mit Religion beschäftigt. Wie zu zeigen sein wird, geht es Smith an dieser Stelle jedoch weit weniger um den persönlichen Glauben als vielmehr um die gesellschaftliche Funktion religiöser Institutionen. Abschließend sollen in einem Fazit die wesentlichen Eckpfeiler des deistischen Grundverständnisses Smiths in ihrer Bedeutung insbesondere für seine ökonomische Theorie nochmals zusammengefasst werden.

\title{
2. The „never to be forgotten Francis Hutcheson“
}

Wie sehr Smith von dem Unterricht bei Francis Hutcheson während seiner Zeit als Student in Glasgow geprägt wurde, belegt ein Brief an Archibald Davidson, datiert vom 16. November 1787, den Smith anlässlich seiner Berufung als Lord Rector der Universität Glasgow verfasst:

\begin{abstract}
"No man can owe greater obligations to a Society than I do to the University of Glasgow. They educated me, they sent me to Oxford, soon after my return to Scotland they elected me one of their own members, and afterwards preferred me to another office, to which the abilities and Virtues of the never to be forgotten Dr. Hutcheson had given a superior degree of illustration.“ (Smith 1987 [1977]: 308f.)
\end{abstract}

Als Professor für Moralphilosophie in Glasgow folgt Smith nicht nur in der Einteilung seiner Vorlesungen seinem akademischen Lehrer, sondern übernimmt auch wesentliche Gedanken Hutchesons. Insbesondere in seinen Ausführungen zur Moralphilosophie folgt Smith dessen Sicht, dass der Mensch nicht rein eigennützig handle, sondern, dank der weisen Einrichtung der Natur, durch seine Gefühle dazu bestimmt ist, das Wohlergehen seiner Mitmenschen in seinem Handeln mit zu berücksichtigen. Der Mensch besitze von Natur aus die Fähigkeit, moralisch gutes Handeln zu erkennen und zu beurteilen (vgl. Hutcheson 1986 [1725]: 5f.). Dabei geht Hutcheson, wie später auch Smith, von der Annahme aus, dass wir die moralische Qualität unseres Handelns am objektiven Urteil unserer Mitmenschen messen können, die unser Verhalten billigen oder missbilligen (vgl. u.a. Hutcheson 1986 [1725]: 42; 87). Diese Art des Urteils ist unabhängig von den damit verbundenen persönlichen Vorteilen, die für uns oder für andere aus dieser Tat resultieren (vgl. Hutcheson 1986 [1725]: 25f.); wir verabscheuen ein Verbrechen auch dann, wenn wir persönlich davon profitieren würden (vgl. Hutcheson 1986 [1725]: 20f.). Wenngleich Smith weniger optimistisch hinsichtlich der „Unbestechlichkeit“ des menschlichen Urteilsvermögens ist als Hutcheson ${ }^{4}$ und vorsichtiger als Hutcheson nicht von einem naturgegebenen „moralischen Sinn“ (vgl. u.a. Hutcheson 1986 [1725]: 29; 34), sondern von sympathetischen Gefühlen ${ }^{5}$ als

4 Vgl. hierzu das in der Auflage letzter Hand der TMS neu eingefügte Kapitel I, iii, 3: „Über die Verfälschung unserer ethischen Gefühle, die aus diesem unseren Hang entsteht, die Reichen und Großen zu bewundern..."

5 Gemeint ist die Fähigkeit, sich in die Lage des anderen hineinzuversetzen, was es dem Betrachter erlaubt, auf dieser Basis die Angemessenheit des Verhaltens aus Zuschauerperspektive moralisch beurteilen zu können. Dabei ist für Smith das moralische Urteil zwar durch unsere Gefühle bestimmt, jedoch erfolgt es nicht, wie bei Hutcheson, unmittelbar auf Basis eines „Moral- 
Grundlage unseres sittlichen Handelns und Urteilens ausgeht (vgl. Smith 1985a [1759]: $\mathrm{I}, \mathrm{i}, 1)$, bleiben die Grundannahmen über das moralische Urteilsvermögen weitgehend gleich.

(1) Basis der Moralphilosophie sowohl Francis Hutchesons wie auch Adam Smiths ist die Annahme, dass der Mensch von Natur aus dazu veranlagt ist, Mitgefühl und generelles Wohlwollen gegenüber seinen Mitmenschen zu empfinden und an ihrer Freude und an ihrem Kummer Anteil zu nehmen. So ist Hutcheson darum bemüht zu zeigen, „dass die menschliche Natur ein elementares uneigennütziges Streben nach dem Glück anderer besitzt...“ (Hutcheson 1986 [1725]: 46). Diese „Veranlagung unserer Natur, das Wohl anderer zu erstreben“ ist für Hutcheson ein „Instinkt, der allen Gründen des Interesses vorausgeht und der uns zur Liebe für andere veranlasst..." (Hutcheson 1986 [1725]: 55). Hingegen können „Begierden, welche zwar auf die Wohlfahrt anderer gerichtet sind, aber, ohne alle andere Neigung, den Begierden nach unserem eigenen Vorteil unterwürfig sein müssen, nichts tugendhaftes in sich haben“ (Hutcheson 1756 [1755]: 97). Wohlwollen und Sympathie sind in seinen Augen die naturgegebene Basis menschlicher Moral und das, was den Menschen vom Tier unterscheidet (vgl. Hutcheson 1756 [1755]: 460f.).

„Diese Sympathie scheint sich in allen unsern Neigungen und Leidenschaften zu äußern. Sie scheinen sich alle[n] andern mitzuteilen. Wir sind nicht nur traurig mit den Betrübten, wir freuen uns nicht nur mit den Glücklichen, sondern auch die Verwunderung, oder das Erstaunen, welches sich an jemanden äußert, erregt eine ähnliche Bewegung der Seele in allen, die ihn sehen“" (Hutcheson 1756 [1755]: 67).

Uneigennütziges Wohlwollen gegenüber anderen ist dabei weder etwas, was auf Selbstliebe oder eine prüfende Vernunft zurückgeführt werden kann noch in irgendeiner Weise von uns willentlich hervorgerufen werden kann. Vielmehr ist diese Fähigkeit dem Einfluss „der göttlichen Gesetze auf unsere Neigungen“ geschuldet (Hutcheson 1756 [1755]: 100). Und obwohl unser objektives moralisches Urteil diese Art des uneigennützigen Wohlwollens gutheißen mag und wir uns hiervon auch göttliche Belohnung versprechen mögen, kann dies uns nur zu dem „Wunsch veranlassen, diese Neigungen zu besitzen, und ihnen gemäß zu handeln“" (Hutcheson 1756 [1755]: 100).

Auch für Smith stellen die Fähigkeit zur Anteilnahme und der uneigennützige Wunsch, das Wohlergehen anderer zu befördern, den Ausgangspunkt seiner moralphilosophischen Überlegungen dar. Gleich zu Beginn der TMS heißt es:

„Man mag den Menschen für noch so egoistisch halten, es liegen doch offenbar gewisse Prinzipien in seiner Natur, die ihn dazu bestimmen, an dem Schicksal anderer Anteil zu nehmen, und die ihm selbst die Glückseligkeit dieser anderen zum Bedürfnis machen, obgleich er keinen anderen Vorteil daraus zieht, als das Vergnügen, Zeuge davon zu sein" (Smith 1985a [1759]: 1).

Obwohl Hutcheson in seinen Schriften in weit stärkerem Maße als Smith in religiösen Dingen Stellung bezieht, betont er an dieser Stelle doch ausdrücklich, dass unser Stre-

sinnes“, sondern es bedarf gleichwohl auch der Reflexion und Interpretation aller Umstände, die das Verhalten beeinflussen (vgl. Griswold 1999: 192). 
ben nach tugendhaftem Handeln nicht auf „Religion gegründet“ sei oder gar dem selbstsüchtigen Wunsch entspringe „die Gottheit werde uns dafür belohnen“ (Hutcheson 1986 [1725]: 25). Denn „Wohltaten von einem Wesen zu erwarten, dessen Interessen von uns unabhängig sind, muss völlig lächerlich sein. Warum sollte Gott Tugend belohnen?“ (Hutcheson 1986 [1725]: 52). Vielmehr glaubt Hutcheson, „dass wir eben durch die Verfassung unserer Natur bestimmt werden, Vergnügen bei der Ausübung der Tugend wahrzunehmen und sie zu billigen, wenn wir selbst oder andere sie ausüben" (Hutcheson 1986 [1725]: 53).

Diese Annahme einer aller christlichen Offenbarung vorausgehenden Fähigkeit zu moralischem Handeln bringt Hutcheson, trotz aller Vorsicht in seinen Formulierungen, ${ }^{6}$ in Konflikt mit der etablierten Amtskirche. Hutcheson, so der Vorwurf der Kirchenleitung, verkünde in seinen Schriften zwei falsche Wabrheiten (vgl. Rae 1895: 12f.): Zum einen behaupte er, dass der Mensch von Natur aus dazu fähig sei, eine Vorstellung von Gut und Böse zu entwickeln noch bevor er von Gott weiß. Zum anderen gehe er davon aus, dass der Maßstab für die moralische Qualität unseres Handelns die Beförderung des Glücks unserer Mitmenschen sei und nicht notwendig die Übereinstimmung mit den Geboten Gottes. Dieser Vorwurf mündet 1738, noch während der Studienzeit Adam Smiths in Glasgow, zu einer offiziellen Anklage wegen Häresie vor der Universitätsleitung, die jedoch, nicht zuletzt wegen des Eintretens der Studierenden für ihren Lehrer, letztlich abgewiesen wird (vgl. Kennedy 2011: 390). Allerdings mag dieser Vorfall die auffällige Zurückhaltung Smiths erklären, hinsichtlich seiner eigenen religiösen Überzeugung klar Stellung zu beziehen.

(2) Auch in Bezug auf die Selbstliebe legt Hutcheson die Grundlage des Smithschen Denkens. Grundsätzlich entspringt die Selbstliebe dem Gebot der Klugheit, unsere eigenen Interessen zu schützen, und nicht dem Wunsch, anderen hierdurch zu schaden. „Lässt uns Hass zu Gegnern jener werden, deren Interessen gegen die unseren stehen, dann ist er nur das Produkt der Selbstliebe und nicht das einer uneigennützigen Bosheit" (Hutcheson 1986 [1725]: 47; vgl. auch 1756 [1755]: 188f.). Selbstliebe wird in Hutchesons System ebenso wenig verleugnet wie in Smiths Theorie. So erstreckt sich unser Wohlwollen zwar in gewissem Sinne auf die gesamte Menschheit, aber nur „sofern kein Interesse dazwischentritt, das es durch Selbstliebe hindert“ (Hutcheson 1986 [1725]: 58) und soweit die Interessen der anderen unserer eigenen Glückseligkeit nicht im Wege stehen (vgl. Hutcheson 1756 [1755]: 53). Grundsätzlich tendiert das von Selbstliebe motivierte Handeln für Hutcheson jedoch dazu, innerhalb der göttlichen Ordnung zum Wohle der Gesamtheit wirksam zu werden:

„Unsere Vernunft kann tatsächlich gewisse Grenzen entdecken, innerhalb derer wir nicht nur aus Selbstliebe und in Übereinstimmung mit dem Wohl des Ganzen handeln können, sondern wo es vollkommen notwendig für das Wohl des Ganzen ist, dass jeder Mensch in diesen Grenzen derart zu seinem eigenen Wohle handelt. Der Mangel solcher Selbstliebe wäre allgemein schädlich. Derjenige also, der sein persönliches Wohl mit der Absicht ver-

6

So schreibt Hutcheson (1986 [1725]: 8) in seinem Vorwort zum „Ursprung unserer Ideen von Schönheit und Tugend“, dass er hoffe, dass in dieser Schrift „niemand etwas entdecken werde, das im Gegensatz zur Religion oder den guten Sitten steht.“ 
folgt, auch zu jener Ordnung beizutragen, die das Wohl des Ganzen wirkt, und mehr noch derjenige, der sein eigenes Wohl mit direktem Blick darauf mehrt, sich selbst zu befähigen, [...] der Menschheit Gutes zu tun, derjenige handelt nicht nur untadelig, sondern ehrenvoll und tugendhaft" (Hutcheson 1986 [1725]: 67f.).

Weit weniger als bei Smith kommt hier bei Hutcheson jedoch die Idee einer invisible hand zum Vorschein, die das selbstsüchtige Handeln einzelner zum allgemeinen Guten wendet. Obwohl Hutcheson an anderer Stelle andeutet, dass das Streben der Seele, unsere Wünsche oder die unserer Freunde und Angehörigen zu erfüllen, tendenziell zum langfristigen Glück der Allgemeinheit beiträgt und somit, dank der Wirkmechanismen einer weise eingerichteten Welt, eine positive Wirkung entfaltet (vgl. Hutcheson 1756 [1755]: 54), scheint er doch eher der Meinung zu sein, dass das Glück der anderen vor allem durch unser Wohlwollen als treibender Kraft gefördert würde, wenngleich er zugibt, ,dass der Urheber der Natur zwischen der Befriedigung unserer großmütigen Neigungen und unserem eigenen höchsten Vorteil eine entfernte Verknüpfung gemacht habe“" (Hutcheson 1756 [1755]: 141f.). Obwohl er durchaus auch die negativen Aspekte egoistischen Verhaltens sieht, hält er primär eigeninteressiertes Handeln für zulässig, insofern es durch die Zustimmung objektiv urteilender Zuschauer legitimiert werden kann (vgl. Pesciarelli 1999: 529). Unter Vorwegnahme der kantischen Unterscheidung von pflichtgemäßem Handeln und Handeln aus Pflicht schreibt Hutcheson:

„[D]as Eigeninteresse mindert das Wohlwollen nur dann, wenn die Tat ohne den Gesichtspunkt des Interesses nicht unternommen worden wäre, oder der Handelnde nicht ebenso viel Gutes vollbracht hätte“ (Hutcheson 1986 [1725]: 77).

Allerdings wird das Wohlwollen gegenüber anderen durch unsere Nähe oder Ferne zu unseren Mitmenschen bestimmt. Hier bewirkt die Natur, ähnlich dem Gravitationsprinzip, dass wir uns von den uns näher Stehenden stärker angezogen fühlen und mehr Anteil an ihrem Wohlergehen nehmen als bei denen, die uns fernstehen (vgl. Hutcheson 1986 [1725]: 100f.).

Auch Smith betont den Stellenwert der Selbstliebe, die natürlicherweise dazu führt, dass wir unseren eigenen Interessen stets den Vorzug vor den Interessen der anderen einräumen (vgl. Aßländer 2007: 153f.). Und auch für ihn wird das selbstsüchtige Streben in erster Linie durch das Urteil der Gesellschaft begrenzt:
„Mag es darum auch wahr sein, dass jedes Individuum in seinem Herzen na- turgemäß sich selbst der ganzen Menschheit vorzieht, so wird es doch nicht wagen, den anderen Menschen in die Augen zu blicken und dabei zu geste- hen, dass es diesem Grundsatz gemäß handelt. Jeder fühlt vielmehr, dass die anderen diesen seinen Hang, sich selbst den Vorzug zu geben, niemals wer- den nachfühlen können..." (Smith 1985a [1759]: 123).

Wie West betont, sind Selbstinteresse und Wohlwollen also auch für Smith kein unüberbrückbarer Widerspruch (vgl. West 1976: 99). Beide Gefühlsregungen haben ihren naturgegebenen Platz und wirken gemeinsam als Handlungsmotive zum Wohle der Gemeinschaft. 
Ebenso wie Hutcheson konstatiert Smith ein abnehmendes Wohlwollen gegenüber uns Fernstehenden. ${ }^{7}$ Selbst der phantasiebegabteste Philanthrop sei eben nur begrenzt fähig, sich das Leiden anderer Menschen vorzustellen, die außerhalb seines Sichtkreises leben. Gesetzt den Fall, so Smith, China würde mit all seinen Bewohnern durch ein Erdbeben zerstört, wäre dieser Mensch vielleicht über das Unglück der Bevölkerung betrübt; er würde sich dann aber sogleich wieder seinen Tagesgeschäften zuwenden und nachts trotz des „Untergang[s] von hundert Millionen seiner Brüder mit der tiefsten Seelenruhe schnarchen - vorausgesetzt, dass er diese niemals gesehen hätte..." (Smith 1985a [1759]: 202).

(3) Ein dritter Punkt, in dem Adam Smith den Ansichten Francis Hutchesons folgt, ist der Glaube an eine harmonische Ordnung des Kosmos. In einer vom Schöpfer weise eingerichteten Welt wirken sowohl die Naturkräfte als auch die sozialen Affekte, die das menschliche Zusammenleben bestimmen, stets zum Wohle des Menschen. So ist es die Natur des Menschen, die ihn zu moralischen Gefühlen und zur Anteilnahme am Schicksal seiner Mitmenschen bestimmt. Allerdings betont Hutcheson weit stärker als Smith explizit das Wirken einer wohlwollenden Gottheit:

\begin{abstract}
„Wenn man $[\ldots]$ nach dem forscht, was notwendig wäre, die Menschen zu wohltätigen Handlungen zu veranlassen oder ihnen einen festen Sinn der Pflicht zum Handeln für das Gemeinwohl zu vermitteln, dann wird das ohne Zweifel folgendes sein: ein Gesetz mit Sanktionen muss notwendig sein, das ein höheres Wesen mit genügend Macht, uns glücklich oder elend zu machen, gegeben hat..." (Hutcheson 1986 [1725]: 134).
\end{abstract}

Hutcheson geht also davon aus, dass die Tugenden , unserer Natur von ihrem Schöpfer ursprünglich eingepflanzt“" wurden ,und später durch unsere eigene Pflege gestärkt und gefestigt werden." (Hutcheson 1986 [1725]: 135). Zwar lasse sich die Existenz Gottes und seiner Güte nicht abschließend beweisen, doch gibt es „,reichlich Wahrscheinlichkeit dafür, die sich aus der ganzen Einrichtung der Natur ableitet, welche, soweit wir wissen, ganz klar zum Wohl des Ganzen eingerichtet zu sein scheint...", selbst „die gelegentlichen Übel scheinen die notwendigen Begleitumstände eines Mechanismus' zu sein, der in höchst überwiegendem Maße auf das Gute ausgerichtet ist" (Hutcheson 1986 [1725]: 158).

Ähnlich glaubt auch Smith an eine zum Wohle des Menschen eingerichtete Welt. Handelt der Mensch entsprechend seiner natürlichen, i.e. gottgegebenen Moral, wird er zum „Mitarbeiter der Gottheit“, da er „die Pläne der Vorsehung ihrer Verwirklichung näher bring[t]“" (Smith 1985a [1759]: 251). Allerdings betont Smith weit stärker als Hutcheson die Rolle der natürlichen Ordnung. ${ }^{8}$ So tritt Gott hinter die von ihm geschaffene Ordnung zurück, wobei Smith anerkennt, dass Gott als Schöpfer den

7 Eine ausführliche Diskussion hierzu findet sich bei Paganelli (2010), die die These vertritt, Smith gehe davon aus, dass die natürliche Sympathie, die wir mit den uns Nahestehenden empfinden, bei zunehmender räumlicher Distanz durch eine Art professioneller Selbstbeherrschung, wie sie insbesondere als moralische Grundlage einer Commercial Society von Bedeutung ist, ersetzt würde.

8 Zur unterschiedlichen Verwendung des Begriffs „,natürlich“ bei Smith vgl. Griswold 1999: $312-317$. 
Menschen mit jenen Eigenschaften ausgestattet hat, die der Erfüllung seiner Zwecke am dienlichsten schienen (vgl. Luterbacher-Maineri 2008: 143). Gott, der Author of Nature, schafft so die menschliche Natur als seinen Erfüllungsgehilfen.

Diese abstraktere Sichtweise von der Wirkung Gottes durch die Natur verdankt sich nicht zuletzt auch Smiths Bewunderung für die Naturwissenschaften und insbesondere die Newtonsche Physik (vgl. Aßländer 2007: 13ff.).

„His ultimate objective was to do for moral philosophy what Sir Isaac Newton had done for natural philosophy“" (Evensky 1989: 142).

In seinen Frühschriften bringt Smith seine Bewunderung für die „Newtonsche Methode“, die anhand einfacher Prämissen auf die Wirklichkeit schließt, zum Ausdruck und hält hieran als Forschungsmethode auch in seinem späteren wissenschaftlichen Vorgehen fest (vgl. Hollander 1998: 105f.):

,[] $\mathrm{n}$ the manner of Sir Isaac Newton we may lay down certain principles known or proved in the beginning, from whence we account for the several phenomena, connecting all together by the same chain [...]" (Smith 1985b [1983]: 145f.)

So sind es für Smith das Streben des Menschen nach Wohlstand und die Fähigkeit zur Sympathie, die als Grundprinzipien die gesellschaftliche Ordnung im Gleichgewicht halten (vgl. Berry 2006: 125f.) und gleichermaßen dem Endzweck der Schöpfung, i.e. der menschlichen Glückseligkeit dienen:

„Die Glückseligkeit der Menschen wie die aller anderen vernunftbegabten Geschöpfe scheint das ursprüngliche Ziel gewesen zu sein, das dem Schöpfer der Natur vorschwebte, als er diese Wesen ins Dasein rief. [...] Indem wir $[\ldots]$ den Geboten unseres moralischen Vermögens gemäß handeln, gebrauchen wir gerade das wirksamste Mittel, um die Glückseligkeit der Menschen zu befördern, und man kann also in gewissem Sinne von uns sagen, dass wir Mitarbeiter der Gottheit sind, und dass wir, soweit es in unserer Macht steht, die Pläne der Vorsehung ihrer Verwirklichung näher bringen“" (Smith 1985a [1759]: 250f.).

Die harmonische Ordnung des Weltganzen wird so für Smith sowohl zum ästhetischen Ideal wie auch zur regulativen Idee seiner Moralphilosophie (vgl. LuterbacherMaineri 2008: 242). Allerdings bleibt Smith skeptisch gegenüber metaphysischen Annahmen und scheinbar gesicherten philosophischen Erkenntnissen. Weit stärker als Hutcheson rekurriert er in seiner Moralphilosophie auf die tatsächliche menschliche Natur (vgl. Griswold 1999: 162-170). Allerdings vertraut er darauf, dass diese menschliche Natur - trotz all ihrer Schwächen - Teil der vom Schöpfer weise eingerichteten Natur ist und zum Wohle des Ganzen wirkt. So ist es gerade die hierin zum Ausdruck kommende stoische Sicht einer der Natur innewohnenden Harmonie als Zeichen göttlicher Vernunft, die erneut die Nähe zum aufgeklärten, christlichen Stoizismus seines Lehrers Hutcheson verrät (vgl. u.a. Clarke 2000: 49; Merikoski 2002: 65f.):

„Auf Schritt und Tritt begegnet uns der Autor und Lenker der Natur, der die Mechanik der moralischen Gefühle so eingerichtet hat, dass systemkonformes Handeln in der Regel das größtmögliche Glück des Einzelnen und die Erhaltung der menschlichen Gattung befördert“" (Ballestrem 2001: 88). 


\section{Die prästabilierte Harmonie als Ausdruck der göttlichen Ordnung}

Wenngleich es möglicherweise zu weit geht zu behaupten, dass der „Deismus [...] als Religionsphilosophie der Aufklärung betrachtet werden“ kann (Luterbacher-Maineri 2008: 76), so ist doch das Denken vieler Schottischer Intellektueller zu Zeiten Smiths durch einen zumindest moderaten Deismus geprägt (vgl. u.a. Evensky 1989: 143; Oslington 2012: 432). In dieser Sichtweise wird Gott als Schöpfer der Welt verstanden, der die Natur weise und harmonisch eingerichtet hat und der in dieser harmonischen Weltordnung erkannt werden kann. Sowohl die Gesetze der Natur als auch die Gesetze der Moral sind von Gott zum Wohle des Menschen geschaffen. Dies gilt auch für die religiöse Ordnung, die zum Wohle des Menschen wirkt, da sie dazu beiträgt, die soziale Ordnung aufrechtzuerhalten, und ein auf sittlichen Grundsätzen basierendes Miteinander der Menschen zu ermöglichen (vgl. Fergusson 2007: 5). Diese gewissermaßen stoische Sicht einer natürlichen Ordnung des Kosmos erlaubt es, naturwissenschaftliche Erkenntnisse mit den religiösen Vorstellungen der Zeit zu versöhnen.

Die Bewunderung der auf einfachen Wirkprinzipien beruhenden Naturwissenschaften und der Glaube an eine harmonische Weltordnung führt viele Theologen und Moralphilosophen, und insbesondere Adam Smith, zu der Annahme, ebensolche Grundprinzipien auch innerhalb der gesellschaftlichen Ordnung aufspüren zu können (vgl. Pabst 2011: 110ff.). Dabei offenbart sich für Smith die göttliche Ordnung nicht zuletzt auch in den natürlichen moralischen Gefühlen des Menschen (vgl. LuterbacherMainieri 2008: 143), die als „Statthalter Gottes“ (Smith 1985a [1759]: 250) unsere moralischen Urteile bestimmen. Von Natur aus ist der Mensch, so die Annahme Smiths, sowohl mit der Fähigkeit zur Sympathie als auch mit einem Hang zur Selbstliebe ausgestattet. Beide Affekte stehen für Smith nicht im Widerspruch, da sich beide wechselseitig kontrollieren. So sind es also seine natürlichen Anlagen, die den Menschen zur Moral befähigen, und auch die soziale Ordnung, wie sie sich im System der natürlichen Freiheif' automatisch ergibt, ist ein Produkt der Natur. Treibende Kraft für unser moralisches Streben ist dabei der Wunsch, in unserem Verhalten von anderen gelobt und anerkannt zu werden und uns dieser Anerkennung auch als würdig zu erweisen (vgl. Smith 1985a [1759]: 176). ${ }^{10}$

Einige frühe Smith-Interpreten (vgl. hierzu Oslington 2011: 3f.) sehen angesichts der zahlreichen Verweise auf eine die Natur weise einrichtende Gottheit Smiths Schriften insgesamt als Werke zur natürlichen Theologie. Allerdings scheint diese Sicht eher gewagt, da Smith weder in seiner TMS noch in seinem WN das Thema natürliche Religion ausdrücklich thematisiert. ${ }^{11}$ Auch die umgekehrte Interpretation, Smith habe ledig-

9 Vgl. hierzu u.a. Smith (1990 [1776]: 267; 385; 442; 582); etwas irreführend wird hier ,,natural liberty“ jedoch von Recktenwald uneinheitlich auch mit „persönlicher Freiheit“ (1990: 267) oder „natürliches Recht auf Freiheit“ (1990: 385) übersetzt.

10 Smith geht jedoch auch davon aus, dass äußere Umstände wie staatliche Reglementierung, Bevormundung, Armut und falsche Erziehung die Wirkung unserer natürlichen moralischen Empfindungen in eine falsche Richtung leiten können (vgl. Aßländer 2003: 39-43).

11 Als Professor für Moralphilosophie hatte Smith in Glasgow die Fächer Ethik, Ökonomie, Recht und natürliche Religion zu unterrichten (vgl. Rae 1895: 54f.). Während aus seinen Vorlesungen zu Ethik und Ökonomie seine beiden Hauptwerke hervorgingen und eine Schrift zur 
lich versucht, seine „Darstellung natürlicher Abläufe [dadurch] überzeugender und auch lebendiger" zu gestalten, dass er sie ,als das Werk eines persönlichen Gottes behandelt" habe (Raphael 1991: 48), und seine religiösen Anspielungen müssten daher als „simply a rhetorical flourish, a not to the convention of the time“ (Fleischacker 2005: 45) gesehen werden, scheint angesichts der Bedeutung, der einer weise eingerichteten Natur in Smiths Werk zukommt, wohl zu einfach.

Weitaus zielführender für die Erklärung des Stellenwertes der Religion in Smiths Gesamtwerk scheint es, das differierende Verständnis von Religion, wie es in seinen verschiedenen Schriften zum Ausdruck kommt, näher zu untersuchen. So scheint Smith in seinen frühen Schriften eine eher evolutionäre Sicht der Religion vertreten zu haben (vgl. Evensky 1998): (1) Auf einer ersten, primitiven Entwicklungsstufe dient Religion in archaischen Gesellschaften dazu, dem Menschen Sicherheit zu geben. Die Beziehung zu Gott ist bestimmt durch den Tauschcharakter von Gehorsam und Gnade. Wie ein Kind nimmt auch der Wilde die Gaben eines höheren Wesens an und fürchtet sich vor der Strafe. Die Gottheit steht außerhalb der natürlichen und menschlichen Ordnung und beherrscht die Natur. Kennzeichnend für primitive Völker sei daher der Glaube an die Macht Gottes, über die Natur zu gebieten und die für den Menschen unerklärlichen Naturphänomene hervorzurufen. (2) Auf der Stufe der Philosophie wird die Welt als Teil einer kosmischen Ordnung begriffen, die der Mensch generell zu erfassen in der Lage ist, auch wenn er ihre Funktionsweise im Einzelnen noch nicht durchschaut. Philosophie wird nun zur Wissenschaft, die die Zusammenhänge der Natur zu erklären sucht (vgl. Smith 1982 [1795]: 45). In dieser Sicht ist die Natur göttlichen Ursprungs und zum Wohle der Menschheit eingerichtet. Alles, auch der Mensch, ist Teil desselben Systems und denselben (Natur)Gesetzen unterworfen. Auf Stufe der Institutionalisierung schließlich werden Religion und die auf ihr gründende kirchliche Ordnung als vom Menschen geschaffenes Instrument begriffen, das dazu dient, die Gemeinschaft moralisch zu verbessern. Religion entwickelt sich zur kirchlichen Institution, die für gesellschaftliche Ordnung sorgt und Erziehungsaufgaben übernimmt. Zwar liegt hierin auch ein gewisses Konfliktpotential, da unterschiedliche Glaubensbekenntnisse widerstreiten und einander bekämpfen. Dennoch dient Religion generell der Aufrechterhaltung der öffentlichen Ordnung und der öffentlichen Ruhe.

Insbesondere die beiden letztgenannten Glaubensauffassungen scheinen von Smith selbst vertreten zu werden, wobei er, wie wir noch sehen werden, zumindest in seinen erhaltenen Schriften darum bemüht ist, strikt zwischen dem Glauben an ein höheres Wesen auf der einen und den etablierten Amtskirchen als Institution auf der anderen Seite $\mathrm{zu}$ unterscheiden.

Zahlreiche Autoren haben versucht, aus den Schriften Adam Smiths Aussagen über seine persönliche religiöse Einstellung herauszulesen. Anlass für die langanhaltende Diskussion über Smiths persönliche religiöse Einstellung gab vor allem die in grundlegender Überarbeitung 1790 erschienene sechste Auflage der TMS. Wesentliche Veränderungen beziehen sich vor allem auf die Streichung von Hinweisen auf eine explizit christliche Glaubensauffassung, die Einfügung seines Kapitels „Über die Verfäl-

Jurisprudenz nicht mehr vollendet wurde, scheint Smith jedoch nie beabsichtigt zu haben, auch ein Werk zur natürlichen Religion zu verfassen. 
schung unserer ethischen Gefühle...", die signifikante Kürzung des sogenannten „Sühne-Absatzes“ (Kennedy 2011: 395), in dem es vor allem um die jenseitige Buße für unsere auf Erden begangenen Sünden ging, und eine generell vorsichtigere und unbestimmtere Wortwahl in Bezug auf Glaubensfragen. Weitaus stärker wird demgegenüber die menschliche Natur als kosmische Ordnungsinstanz hervorgehoben; zudem fügt Smith auch einige neue Passagen ein, die eine nicht-christliche, aber sehr wohl deistische Haltung untermauern (vgl. Clarke 2002: 11; Luterbacher-Maineri 2008: 164-168). Gerade hinsichtlich dieses scheinbar zunehmenden Abstandes, den Smith am Ende seines Lebens gegenüber einer explizit christlichen oder gar calvinistischen Glaubensauffassung zu suchen scheint, gehen die Meinungen der Smith-Interpretationen weit auseinander. So etwa sieht Jerry Evensky (vgl. 1998) Smith als tief gläubigen Menschen; dies folge aus der Betonung der natürlichen Ordnung, die auf dem Plan eines wohlwollenden und allwissenden Wesens beruhe. Demgegenüber betonen Ingrid Merikoski (vgl. 2002) und Peter H. Clarke (vgl. 2000) Smiths zunehmende Hinwendung zu einem christlichen Stoizismus, was letztlich in seiner deistischen Weltsicht zum Ausdruck komme, die er gerade in der Ausgabe letzter Hand der TMS nochmals deutlich unterstreiche. Gavin Kennedy (vgl. 2013) hingegen sieht Smith als generell a-religiösen Menschen. Die religiösen Textanspielungen seien daher vor allem dem Umstand geschuldet, dass Smith es vermeiden wollte, das Missfallen der konservativ-religiösen Universitäts- und Gelehrtenkreise zu erregen; er sah sich daher gezwungen, seine kirchenkritische und a-religiöse Haltung hinter einem „Vorhang der Religiosität" (Kennedy 2011: 385) zu verstecken. Auch habe Smith die Rücksichtnahme auf die Gefühle seiner tief-religiösen Mutter dazu veranlasst, wesentliche Änderungen in der TMS erst nach deren Tode im Jahr 1784 vorzunehmen.

Ungeachtet dieses Spektrums möglicher Interpretationen bleibt jedoch festzuhalten, dass trotz aller vorgenommenen Änderungen in der TMS-Ausgabe letzter Hand die prästabilierte Harmonie einer natürlichen Ordnung, die die eigennützigen Bestrebungen des Einzelnen zum Wohle der Gemeinschaft wirksam werden lässt, und die natürliche Fähigkeit des Menschen, Sympathie für seine Mitmenschen empfinden zu können, die beiden zentralen Eckpfeiler in Smiths Schriften darstellen. Beide Annahmen gehen letztlich auf die moralphilosophischen Entwürfe Francis Hutchesons zurück und sind sowohl tragend für Smiths moralische wie auch für seine ökonomischen Vorstellungen. Deutlich wird dies, wenn man die Aussagen zu ökonomischen Sachverhalten, mit denen sich Smith auch in seiner TMS beschäftigt, näher durchleuchtet. Bereits hier entwirft Smith eine Moral, die das Selbstinteresse der Kaufleute als Motor wirtschaftlichen Handelns positiv konnotiert. Nicht wenige Autoren führen diese positive Konnotation materialistischer menschlicher Bestrebungen namentlich auf den Einfluss David Humes zurück (vgl. u.a. Raphael/Macfie 1982: 10ff.; Clarke 2002: 15ff.; Rothschild 2002: 130f.). Jedoch lässt sich zeigen, dass die Vorstellung moderater Eigenliebe als Gebot der Klugheit nicht nur bereits bei Hutcheson angelegt, sondern augenscheinlich auch mit der von ihm propagierten deistischen Weltsicht durchaus kompatibel ist.

Während Hume vor allem die Gier nach Luxus als eigentliches Motiv des Wirtschaftens und das Verlangen nach einem „prachtvollen Leben“ als Motor des Wirtschaftswachstums betont (vgl. 1988a [1741]: 182-186), sieht Smith in diesem Streben nicht 
nur eine Grundkonstante der menschlichen Natur, sondern das Werk einer diese menschliche Natur zu seinem Zwecke nutzenden Gottheit. Ziel dieser Gottheit ist die Vervollkommnung der menschlichen Gemeinschaft und das Wohl des Menschen. Dank einer geschickten Täuschung der Natur führe das Streben des Einzelnen nach materiellem Wohlstand gerade dazu, dass er sich zum Woble der Gemeinschaft anstrengt, während er glaubt, ausschließlich seinen eigenen Interessen zu dienen. Dabei betont Smith ausdrücklich auch den hieraus für die Gemeinschaft resultierenden immateriellen Nutzen:

„Denn diese Täuschung ist es, was den Fleiß der Menschen erweckt und in beständiger Bewegung erhält. Sie ist es, was sie zuerst antreibt, den Boden zu bearbeiten, Häuser zu bauen, Städte und staatliche Gemeinwesen zu gründen, alle die Wissenschaften und Künste zu erfinden und auszubilden, die das menschliche Leben veredeln und verschönern [...]“ (Smith 1985a [1759]: 315).

Diese Täuschung der Natur führt also letztlich dazu, dass die materialistischen Bestrebungen des Menschen, selbst dann, wenn sie über die durch Klugheit gebotene Eigenliebe - die durch Rücksichtnahme auf andere und auf die Gemeinschaftsinteressen gekennzeichnet ist (vgl. Heise 1991: 74) - hinausgehen, dennoch zum Wohle aller wirken. Sie entfaltet ihre Wirkung sowohl in sozialer als auch in ökonomischer Hinsicht, da sie zum einen dafür sorgt, dass die Launen der Reichen Wohlstand für alle erzeugen, da sie gezwungen sind, diese für die geleisteten Dienste zu entlohnen (vgl. Smith 1985a [1759]: 315ff.), und zum anderen dazu beiträgt, dass das Streben nach individuellem Vorteil zur optimalen Ressourcenallokation zum Wohle der Gemeinschaft führt (vgl. Smith 1990 [1776]: 370f.). In dieser Sichtweise ruht das System der natürlichen Freiheit, in dem es das heilige Recht des Einzelnen ist, seine persönlichen Ziele nach Maßgabe seiner eigenen Wünsche und in Kenntnis der ihm hierzu zur Verfügung stehenden Mittel zu verfolgen (vgl. Smith 1990 [1776]: 451), gerade auf dem Fundament einer als harmonisch gedachten kosmischen Ordnung. So ist der Mensch, wie sich dies bereits in Anklängen bei Hutcheson findet, gerade deshalb dazu befähigt in Freiheit zu leben, weil er durch die Fähigkeit zum Respekt gegenüber anderen und seinem Wunsch, das Wohl der anderen und damit der Gemeinschaft zu befördern, hierfür disponiert ist (vgl. Ballestrem 2001: 33f.).

Smith sieht die sich in seiner Zeit entwickelnde Commercial Society in gewisser Weise als gesellschaftlichen Fortschritt, da sie ein Mindestmaß an Gleichheit schafft, feudale Standesunterschiede nivelliert und letztlich zur Wohlstandsverbesserung insbesondere der ärmeren Bevölkerungsschichten beiträgt (vgl. Hanley 2009: 45, FN 43). Er ist daher auf der Suche nach einer mit den ökonomischen Entwicklungen seiner Zeit kompatiblen Moraltheorie. Sowohl in seiner TMS als auch in seinem WN findet er sie in der segensreichen Wirkung, die auch das eigennützige Streben nach materiellem Wohlstand für den Fortschritt der Gemeinschaft entfaltet. So ist es im WN einzig der Bettler, der allein vom Wohlwollen seiner Mitmenschen abhängig bleibt. Wer hingegen für seinen materiellen Wohlstand arbeitet, hat mit dem durch seine Arbeit geschaffenen Produktionsüberschuss ein Tauschäquivalent zu bieten und kann auch ohne das Wohlwollen der anderen überleben (vgl. Smith 1990 [1776]: 17). Wer arbeitet besitzt Würde, er besitzt ein Tauschprodukt und tritt als Geschäftspartner und nicht als Bittsteller auf (vgl. Ballestrem 2001: 144f.). Und erneut ist es eine dem Menschen von 
Natur aus gegebene Fähigkeit, der Hang zu Handel und Tausch (vgl. Smith 1990 [1776]: 16f.), der sich dank der harmonischen Ordnung zum Wohle aller auswirkt.

Allerdings wäre es falsch, hieraus voreilig schließen zu wollen, Smith habe damit einem reinen Laissez-Faire-Kapitalismus den Weg bereiten wollen. Zwar sieht Smith den Menschen bestimmt durch sein Streben nach individuellen Vorteilen und dem Wunsch nach Verbesserung seiner wirtschaftlichen Lage und konnotiert die Wirkung dieses Strebens insgesamt durchaus positiv (vgl. Mehta 2006: 250f.). Aber dieses Streben findet seine Grenze in den sympathetischen Gefühlen gegenüber unseren Mitmenschen, die uns dazu veranlassen, unsere egoistischen Bestrebungen auf ein allgemein verträgliches $\mathrm{Maß}$ zu reduzieren. Dieses, ebenfalls natürliche, Interesse des Menschen am Wohlergehen seiner Mitmenschen, das Smith ebenso wie Hutcheson als Grundlage einer natürlichen Moral annimmt, bildet das wesentliche Korrektiv gegen ein Überhandnehmen des Eigeninteresses, auch in ökonomischen Belangen. Im Spiegel des Urteils der anderen betrachtet der Mensch sein Handeln, und aus Furcht davor, sie könnten sein selbstsüchtiges Handeln nicht billigen, begrenzt er sein eigennütziges Streben (vgl. Aßländer 2007: 141f.).

„In dem Wettlauf nach Reichtum, Ehre und Avancement, da mag er rennen, so schnell er kann, und jeden Nerv und jeden Muskel anspannen, um all seine Mitbewerber zu überholen. Sollte er aber einen von ihnen niederrennen oder zu Boden werfen, dann wäre es mit der Nachsicht der Zuschauer ganz und gar zu Ende. Das wäre eine Verletzung der ehrlichen Spielregeln, die sie nicht zulassen können. Der andere ist für sie in jeder Hinsicht so gut wie dieser; sie stimmen jener Selbstliebe nicht zu, in der er sich selbst zu hoch über den anderen stellt, und sie können die Motive nicht nachfühlen, die ihn bewogen, den anderen zu Schaden zu bringen.“ (Smith 1985a [1759]: 124)

Gerade im System der natürlichen Freiheit findet der Egoismus des Einzelnen stets in der Furcht vor dem Urteil des unparteiischen Zuschauers seine Schranken. Wenn wir hingegen glauben, Eigennutz und Selbstliebe seien die einzigen Antriebe unseres Handelns, so liegt das, wie insbesondere Francis Hutcheson betont, an einer falschen Erziehung des Menschen und daran, dass uns von „der Kanzel, den Schulen [...] und den durch sie beherrschten Unterredungen her der Begriff der Selbstliebe als das einzige Motiv zum Handeln eingeprägt worden ist" (Hutcheson 1986 [1725]: 55). Auch das wirtschaftliche Handeln unterliegt dem Urteil eines unparteiischen Zuschauers und bleibt so in allgemeine Vorstellungen von Moral und Gerechtigkeit eingebunden (vgl. Aßländer 2007: 129). Sowohl der systematisch wirkende Ausgleichsmechanismus der invisible hand als auch die qua moralischer Gefühle geäußerte Zurückhaltung des Einzelnen in seinem selbstsüchtigen Streben sind für Smith Teil der von der Gottheit weise eingerichteten Natur.

\section{Glaubensfreiheit und der Markt der Religionen}

Grundsätzlich scheint Smith in seinem Werk zwischen zwei unterschiedlichen Sichtweisen auf Religion zu unterscheiden. Zum einen ist dies der Glaube an einen Schöpfergott, der die Welt weise und zum Wohle der Menschheit eingerichtet hat. Zwar ist Smith darum bemüht, eine Wortwahl zu finden, die diese Auffassung nicht unmittelbar mit einem bestimmten religiösen Bekenntnis in Zusammenhang bringt, etwa dann, 
wenn er den „unparteiischen Zuschauer“ als „Tribunal [des] eigenen Gewissens“ (Smith 1985a [1759]: 194) bezeichnet, ohne dabei jedoch einen direkten Bezug zur religiösen Offenbarung herzustellen. Doch durchzieht die Vorstellung einer vom Schöpfer weise eingerichtete Natur seine beiden zentralen Schriften und ist wesentlich vom Denken seines Lehrers Francis Hutcheson beeinflusst.

Strikt zu trennen hiervon ist, zum anderen, Smiths Einschätzung der Amtskirchen, die in Form bestimmter Bekenntnisse und Konfessionen als religiöse Institution begriffen werden müssen und auf ihre Funktion für die Gesellschaft hin untersucht werden können. Wie andere gesellschaftliche Institutionen auch - z.B. Bildungseinrichtungen oder Institutionen der Rechtspflege - dienen Kirchen als Institution dabei einem gesellschaftlichen Zweck und können hinsichtlich ihrer Zweckerreichung optimiert, d.h. verbessert werden.

Dies erklärt, warum Smith das Thema Kirche unter der Überschrift „Ausgaben für Bildungseinrichtungen für Menschen jeden Alters“ im Kapitel „Öffentliche Ausgaben" des WN diskutiert. Hier geht es ihm nicht um die Frage göttlichen Wirkens in der Welt, sondern um die Frage nach der optimalen Ausgestaltung der Kirche als Institution in einer an den Ideen persönlicher Freiheit und Toleranz ausgerichteten Wirtschafts- und Gesellschaftsordnung. Als Bildungsstätte ist es die Aufgabe der Kirchen, für die religiöse Unterweisung der Bürger zu sorgen und damit ihren Beitrag zur Aufrechterhaltung der öffentlichen Ordnung zu leisten (vgl. Smith 1990 [1776]: 668). Zentral ist für Smith daher die Frage nach der optimalen Ausgestaltung dieser Bildungseinrichtungen zum Nutzen der Gläubigen. Dabei stehen Fragen der Finanzierung, Anreize zur Arbeitsmotivation der Kirchenbediensteten und die Verhinderung potentiellen Machtmissbrauchs durch eine Staatskirche im Zentrum seiner Betrachtungen. Wie bei anderen Bildungseinrichtungen auch plädiert Smith für einen Konkurren₹markt, auf dem verschiedene Religionsanbieter um die Gunst der Gläubigen konkurrieren. Nur dann, so glaubt Smith, wenn es keiner der Konfessionen gelänge, eine marktbeherrschende Stellung zu erlangen, könne religiöser Fanatismus verhindert werden, da sich nun alle Konfessionen in derselben Lage befänden und gezwungen wären, einander zu tolerieren (vgl. Smith 1990 [1776]: 672ff.).

Zugleich sieht Smith es als Aufgabe der Kirche, ihren Beitrag zur Aufrechterhaltung der öffentlichen Ordnung zu leisten, wobei er jedoch hinsichtlich der Frage, ob dieser Beitrag wirksamer von einer staatlichen oder einer staatlich unabhängigen Kirche geleistet werden könne, unentschieden bleibt (vgl. Smith 1990 [1776]: 677-690). Während er zum einen den Amtsmissbrauch der etablierten Staatskirchen fürchtet, die dazu neigten, sich mit den Herrschern zu verbünden, um sich im Schutz durch die Obrigkeit mehr um ihr Einkommen als um ihre Gläubigen zu kümmern, beunruhigt ihn zum andern aber auch der Fanatismus der kleinen Sekten, die in ihrem Werben um Anhänger dazu tendierten, radikale Positionen zu vertreten. Um den Arbeitseifer der Geistlichen für ihre Gemeinden zu befördern, empfiehlt Smith, deren Gehälter mindestens partiell aus den Mitteln der Gemeinde zu bestreiten. Ansonsten durchaus skeptisch gegenüber den Einrichtungen der katholischen Kirche, bemerkt Smith hier mit einer gewissen Bewunderung, dass sich die Bettelorden der katholischen Kirche ausschließlich aus den Spenden ihrer Gemeinde finanzierten, was ihren Diensteifer be- 
trächtlich steigern würde: „Es ist mit ihnen wie mit den Husaren und der leichten Infanterie in manchen Armeen: Keine Beute, keine Einnahme" (Smith 1990 [1776]: 669).

Auch hier existieren in der neueren Literatur zahlreiche Interpretationsversuche, die vor allem darauf abzielen, Smiths Konkurrenz der Religionen in das Konzept einer liberalen Wirtschaftsordnung einzuordnen. (1) So glaubt beispielsweise Gary Anderson (vgl. 1988), hierin erste Anzeichen eines Ökonomischen Imperialismus avant la lettre ausmachen zu können. Indem Smith die Grundsätze ökonomischer Rationalität auf das Problem der optimalen Ausgestaltung kirchlicher Institutionen anwendet, lege er gleichsam das Konzept einer „Economics of Religion“ (Anderson 1988: 1066) vor. So diskutiere Smith unter anderem ökonomische Anreize, die den Einzelnen dazu veranlassen, einer bestimmten Religionsgemeinschaft beizutreten. Die durch eine gottgefällige Lebensführung erlangte Reputation besitzt für den Gläubigen einen Marktwert, und dies bilde das ökonomische Hauptmotiv für den Beitritt zu einer Kirchengemeinde. ${ }^{12}$ $\mathrm{Da}$ die Glaubensgemeinschaften in ihrer Gesamtheit für die Reputation ihrer Mitglieder haften, seien sie stets darum bemüht, auf die moralisch untadelige Lebensführung ihrer Mitglieder zu achten (vgl. Anderson 1988: 1071-1075). Insbesondere kleine Religionsgemeinschaften hätten daher ein massives Interesse an der Aufrechterhaltung der Gruppenmoral ihrer Mitglieder (vgl. Smith 1990 [1776]: 675). - Eine These wie sie später auch von Max Weber in seiner religionssoziologischen Untersuchung der protestantischen Sekten geäußert wird (vgl. Weber 1988 [1906]). Da zudem ein staatliches Kirchenmonopol religiöse Intoleranz fördern würde, könne nur ein System freier Konfessionen den sektiererischen Eifer der verschiedenen Kirchen qua Leistungswettbewerb abschwächen. Auch sähe Smith im Glauben an ein höheres Wesen und die Möglichkeit der göttlichen Strafe für menschliches Unrecht eine wirksame Möglichkeit, die Bemühungen irdischer Autoritäten um die Aufrechterhaltung der gesellschaftlichen Ordnung zu unterstützen (vgl. Anderson 1988: 1069).

(2) Demgegenüber betonen Leathers und Raines (vgl. 1992; 2008) primär den gesellschaftlichen Nutzen, der mit dieser Etablierung eines Markts der Religionen einhergeht. Durch einen begrenzten, d.h. unter staatlicher Aufsicht stattfindenden Wettbewerb würde die Übermacht einer Konfession verhindert (vgl. Leathers/Raines 1992: 503f.). Zugleich könne der Staat als Aufsichtsorgan dazu beitragen, religiösen Fanatismus zu mildern und die ordentliche Amtsführung des Klerus zu überwachen, beispielsweise indem er durch Zulassungsprüfungen bei der Besetzung kirchlicher Ämter für eine geeignete Kandidatenauswahl sorge. Auch könne der Staat durch eine Regulierung der Besoldung dafür Sorge tragen, dass die Ausbildungskosten für das Erlernen eines geistigen Berufes bezahlbar blieben und durch eine angemessene Entlohnung der Geistlichen dazu beitragen, die Würde des Amtes aufrechtzuerhalten. Aufgabe derartiger staatlich geprüfter Kirchen sei es dann, für öffentliche Ruhe und Gehorsam gegenüber der Obrigkeit zu sorgen. Wichtig sei zudem, dass durch dieses regulierte Oligopol eine hinreichende Vielfalt an unterschiedlichen Glaubensbekenntnissen existieren

12 Auch in der TMS geht Smith davon aus, „dass die Menschen im allgemeinen geneigt sind, großes Vertrauen in die Rechtschaffenheit jener Menschen zu setzen, die ein tiefes religiöses Empfinden besitzen“ (Smith 1985a [1759]: 258), bringt diese Aussage aber in keinerlei institutionellen Kontext. 
würde, die es jedem Gläubigen erlaubten, die für ihn und seine Bedürfnisse passende Religionsgemeinschaft zu wählen (vgl. Leathers/Raines 2008: 357f.). Smith plädiere daher für keine atomistische Konkurrenz der Religionen, sondern - ökonomisch gesprochen - für ein weites Oligopol unter staatlicher Aufsicht.

(3) Eine andere Interpretation stammt von Ekelund et al. (vgl. 2005). Sie sehen Smiths Plädoyer für Religionsfreiheit als Ausdruck einer von Smith geforderten Konsumentensouveränität. Die Möglichkeit, zwischen verschiedenen Religionsgemeinschaften wählen zu können, stärke die Position der Gläubigen. Dies sei jedoch nur möglich, wo eine starke zwischenkirchliche und innerkirchliche Konkurrenz einen Leistungswettbewerb unter den Anbietern garantiere. Kirchenmonopole hingegen würden eine nur mäßige Versorgung der Bevölkerung garantieren, da hier der Klerus mit den Herrschenden paktiere und nur wenig Interesse für die unteren Bevölkerungsschichten zeige, und zugleich die staatliche Ordnung bedrohen, da es ihre privilegierte Stellung der Kirche erlaube, mit dem Staat um Macht und Steuereinnahmen und den Anspruch auf Rechtsprechung zu konkurrieren. Ausdrücklich betone Smith zudem den Nutzen einer leistungsorientierten Entlohnung (vgl. Smith 1990 [1776]: 609). Während unterbezahlte Amtsträger dazu tendierten, ihre Dienste nur nachlässig zu versehen, da sie ihr Auskommen anderweitig suchen müssten, führe Überbezahlung zu Ausschweifungen und habe damit ebenfalls eine Vernachlässigung der Dienstpflichten zur Folge (vgl. Smith 1990 [1776]: 693). Da Religion kein homogenes Gut sei und jeder Gläubige andere Bedürfnisse habe, die er durch die Ausübung seiner Religion zu befriedigen sucht, erweise sich aus Sicht der Konsumenten ein zwischenkirchlicher Wettbewerb als vorteilhaft, da er eine Zunahme des Angebots fördere. Allerdings führe der innerkirchliche Wettbewerb bei der Besetzung von Kirchenämtern mitunter zu einer Radikalisierung der Geistlichen, die, um Aufmerksamkeit zu erregen, leicht zum Fanatismus neigen und ihre Kirchengemeinden zu spalten drohen. Entsprechend plädiere Smith für eine Mischform aus Staatskirche und Mitsprachrechten der jeweiligen Gemeinden.

Das hier angeführte Spektrum der Interpretationen zeigt die Schwierigkeiten, die mit der Deutung des Smithschen Konzepts des Markts der Religionen einhergehen. Weitaus konsequenter als Hutcheson, der ebenso wie Smith den religiösen Eifer der Sekten verurteilt (vgl. Hutcheson 1986 [1725]: 92f.), betont Smith die Rolle der Amtskirchen als Erzziebungseinrichtung und wohlweislich nicht als Verkünder von Wabrheit. Er erkennt die Bedeutung, die die etablierten Kirchen für die moralische Unterweisung der Bevölkerung und für die Aufrechterhaltung der öffentlichen Ordnung haben, an, doch lässt sich zwischen den Zeilen erkennen, dass Smith dem exklusiven Anspruch der jeweiligen Kirchen misstraut, je für sich den Weg zum Seelenheil weisen zu können. So ist er auch an dieser Stelle darum bemüht, jenseits einer objektiven Diskussion der möglichen Ausgestaltung derartiger Institutionen, keinerlei persönliche Präferenzen für eine der Kirchen zu äußern.

Während Smith in seiner TMS der Diskussion religiöser Bekenntnisse gezielt auszuweichen scheint und auf das Gebot der religiösen Toleranz nicht eigens eingeht, scheint ihm im Wealth of Nations der Pluralismus kirchlicher Institutionen aus ökonomischer Sicht mindestens der Analyse wert. Zwar ließe sich werksgeschichtlich mit Luterbacher-Maineri (vgl. 2008: 165f.) argumentieren, dass Smith bei der Abfassung seines WN nicht mehr in universitären Diensten stand, sich als Wissenschaftler bereits 
einen Namen gemacht hatte und daher weit freier in seiner Argumentation war. Allerdings trifft dies auch für die überarbeiteten Versionen seiner TMS zu, insbesondere für die 1790, kurz vor seinem Tod erschienene, gründlich überarbeitete sechste Auflage. Wie immer man also die persönliche religiöse Einstellung Adam Smiths interpretieren mag - ob tief religiös, gemäßigt deistisch oder als aufgeklärten Stoizismus - so lässt sich doch, zumindest gegenüber den etablierten Amtskirchen seiner Zeit, eine gewisse kirchenkritische, wenngleich keine grundsätzlich glaubenskritische oder gar atheistische Haltung konstatieren (vgl. Raphael 2007: 104).

\section{5. $\quad$ Fazit}

Wenngleich die verschiedenen, mitunter widersprüchlichen Äußerungen zu religiösen Fragen im Gesamtwerk Smiths Raum für unterschiedlichste Interpretationen geben, wird man Smiths Schriften wohl auch vor dem Hintergrund seiner Zeit und als Produkt der historischen Gegebenheiten betrachten müssen (vgl. Clarke 2000: 50). Smith ist geprägt durch die Erfahrungen seiner Jugend und den konservativen Geist der englischen und schottischen Universitäten, die eine aufgeklärte Geisteserhaltung erschweren. Als Student erlebt er die Anfeindungen Hutchesons durch die presbyterianische Kirche und seine Anklage wegen Häresie. Seine Freundschaft mit dem als Atheisten geltenden David Hume bringt ihn selbst mehrfach in Verlegenheit (vgl. Rae 1895: 125; Ross 1998: 429-433). Ein auf Hume verfasster harmloser Nachruf, in dem er dessen ruhiges Dahinscheiden schildert, bringt Smith selbst in den Verdacht des Atheismus, da er, so der Präsident des Magdalenen Colleges in Oxford, die Welt glauben machen wolle, dass Atheismus das wirksamste Mittel sei, um die Furcht vor dem Tod zu bekämpfen (vgl. Rae 1895: 311ff.). Smith ist in seinen Schriften daher bemüht, auch nicht den kleinsten Hinweis darauf zu geben, er würde selbst eine atheistische Philosophie vertreten oder Grundsätze behaupten, die mit den Glaubenswabrbeiten der etablierten Kirche unvereinbar sind. Doch vergebens: Gerade seine in der sechsten Auflage der TMS vorgenommenen Änderungen und der darin angeblich zum Ausdruck kommende zunehmende Abstand zur christlichen Glaubenslehre stoßen bei den Zeitgenossen auf Kritik und werden erneut dem schlechten Einfluss des zum Zeitpunkt der Veröffentlichung bereits verstorbenen Hume angelastet (vgl. Raphael 2007: 100ff.).

Dennoch scheint Smith in seinem Werk weit weniger von seinem religionskritischen Freund Hume - wie dies bereits einige seiner Zeitgenossen behaupteten - als von seinem Lehrer Francis Hutcheson beeinflusst. Zwar folgt Smith Hume in der Auffassung, dass es das Streben nach materiellem Wohlstand sei, das uns in wirtschaftlichen Belangen zum Handeln bewege (vgl. insb. Hume 1988a [1741]; 1988b [1741]), aber der hier bei Smith zum Vorschein kommende Eigennutz als Handlungsmotiv trägt doch weit eher die Züge der am Gebot der Klugheit orientierten Eigenliebe, wie sie auch Hutcheson dem Menschen in seiner Moralphilosophie grundsätzlich zugesteht.

Auch in seinem unverbrüchlichen Glauben an die durch eine Gottheit wohlgeordnete Natur - egal welche religiösen Bezüge man hier unterstellen mag - folgt Smith konsequent den Ansichten Hutchesons, wenngleich er, was möglicherweise seiner Bewunderung für Newton geschuldet sein mag, hier einen wesentlich mechanischeren Ansatz 
wählt: So ist es eben nicht allein das Wohlwollen gegenüber unseren Mitmenschen, das uns zum Handeln bewegt, sondern auch die Furcht der Missbilligung durch den unparteiischen Zuschauer. Und selbst da, wo unser Handeln scheinbar ausschließlich unseren eigenen Interessen dient und wir die Stimme des unparteiischen Zuschauers ignorieren, schafft die invisible hand auf wundervolle Weise einen gerechten Ausgleich (vgl. Aßländer 2003: 52f.). So ersetzt Smith, wie Luterbacher-Maineri (2008: 331) es formuliert, sein ,eigentlich pessimistisches Menschenbild [...] durch sein optimistisches Weltbild.“ Wenngleich man darüber spekulieren mag, ob die Metapher der unsichtbaren Hand selbst lediglich eine Reminiszenz an den religiösen Sprachgebrauch der Zeit ist (vgl. Raphael 1991: 48f.) oder eher einen ironischen Seitenhieb auf die klerikalen Gelehrten Glasgows darstellt (vgl. Rothschild 2002: 130), ist sie doch Ausdruck eines Glaubens an die dem Kosmos innewohnende prästabilierte Harmonie, den Smith von seinem Lehrer Hutcheson übernimmt.

Unabhängig hiervon sieht Smith die Institutionalisierung von Religion in Form der Amtskirche als eine neben anderen Einrichtungen zur Volkserziehung, die sich ungeachtet der verkündeten theologischen Glaubenswahrheiten neutral auf Grundlage ihrer Nützlichkeit für die Gemeinschaft analysieren lässt. Hier empfiehlt Smith den Kirchen Toleranz gegenüber anderen Glaubensgemeinschaften, nicht weil dies notwendig seiner religiösen oder politischen Überzeugung entspräche, sondern weil dies in seinen Augen für die Entwicklung der Gesellschaft förderlich ist. So gesehen ist die berühmt gewordene Stelle des Markts der Religionen in seinem WN weder einem ökonomischen Imperialismus avant la lettre noch - lässt man den durchaus spürbaren kirchenkritischen Unterton außer Acht - ein ökonomisch verbrämtes Plädoyer für religiöse Toleranz. Vielmehr geht es Smith um eine möglichst vollständige Untersuchung des gesellschaftlichen Nutzens und der möglichst effizienten Ausgestaltung staatlicher Bildungseinrichtungen. Damit ist Kirche für Smith exakt das als was er sie einführt: eine „Bildungseinrichtung für Menschen jeden Alters“, die auf ihren möglichen Beitrag zur Förderung des Wohlstands der Nationen hin untersucht werden muss.

\section{Literaturverzeichnis}

Anderson, G. M. (1988): Mr. Smith and the Preachers: The Economics of Religion in the Wealth of Nations, in: Journal of Political Economy, Vol. 96/No. 5, 1066-1088.

Aßländer, M. S. (2003): Adam Smith, Moralphilosophie und Ökonomie in kritischer Absicht, in: Breuer, M./Brink, A./Schumann, O. J. (Hrsg.): Wirtschaftsethik als kritische Sozialwissenschaft, Bern: Haupt, 25-61.

Aßländer, M. S. (2007): Adam Smith zur Einführung, Hamburg: Junius.

Ballestrem, K. (2001): Adam Smith, München: C. H. Beck.

Berry, C. J. (2006): Smith and Science, in: Haakonssen, K. (Ed.): The Cambridge Companion to Adam Smith, Cambridge: Cambridge University Press, 112-135.

Brentano, L. J. (1877): Das Arbeitsverhältnis gemäß dem heutigen Recht, Leipzig 1877. Nachdruck: Goldbach: Keip Verlag. 
Büscher, M. (1991): Gott und Markt - religionsgeschichtliche Wurzeln Adam Smiths und die „Invisible Hand“ in der säkularisierten Industriegesellschaft, in: MeyerFaje, A./Ulrich, P. (Hrsg.): Der andere Adam Smith - Beiträge zur Neubestimmung von Ökonomie als Politischer Ökonomie, Bern: Haupt Verlag, 123-144.

Clarke, P. H. (2000): Adam Smith, Stoicism and Religion in the 18th Century, in: History of the Human Science, Vol. 13/No. 4, 49-72.

Clarke, P. H. (2002): Unity in the Influences of Adam Smith, in: History of Economics Review, No. 36, 10-25.

Eckstein, W. (1985): Einleitung des Herausgebers, in: Smith, A.: Theorie der ethischen Gefühle, Hamburg: Felix Meiner, XI-LXXI.

Ekelund, R. B. Jr./Hébert, R. F./Tollison, R. D. (2005): Adam Smith on Religion and Market Structure, in: History of Political Economy, Vol. 37/No. 4, 647-660.

Evensky, J. (1989): The Evolution of Adam Smith's View on Political Economy, in: History of Political Economy, Vol. 21/No. 1, 123-145.

Evensky, J. (1998): Adam Smith's Moral Philosophy: The Role of Religion and Its Relationship to Philosophy and Ethics in the Evolution of Science, in: History of Political Economy, Vol. 30/No. 1, 17-42.

Fergusson, D. (2007): Introduction, in: ders.: (Ed.):. Scottish Philosophical Theology 1700-2000, Exeter: Imprint Academic, 1-24.

Fleischacker, S. (2005): On Adam Smith's Wealth of Nations, Princeton: Princeton University Press.

Griswold, C. L. jr. (1999): Adam Smith and the Virtues of Enlightenment, Cambridge: Cambridge University Press.

Haakonssen, K. (2006): The Coherence of Smith's Thought, in: ders. (Ed.): The Cambridge Companion to Adam Smith, Cambridge: Cambridge University Press, 1-21.

Hanley, R. P. (2009): Adam Smith and the Character of Virtue, Cambridge: Cambridge University Press.

Heise, P. A. (1991): Stoicism in Adam Smith's Model of Human Behavior, in: Jahrbuch für Ökonomie und Gesellschaft, Bd. 9: Adam Smiths Beitrag zur Gesellschaftswissenschaft, Frankfurt a.M.: Campus, 64-78.

Hollander S. (1998): Adam Smith and the Self-Interest Axiom, in: ders.: The Literature of Political Economy, Collected Essays II, London: Routledge.

Hume, D. (1988a [1741]): Über Handel, in: ders.: Politische und ökonomische Essays, Bd. 2., Bermbach, U. (Hrsg.), Hamburg: Felix Meiner.

Hume, D. (1988b [1741]): Über Verfeinerung in den Künsten, in: ders.: Politische und ökonomische Essays, Bd. 2, Bermbach, U. (Hrsg.), Hamburg: Felix Meiner.

Hutcheson, F. (1756 [1755]): Sittenlehre der Vernunft (A System of Moral Philosophy), Bd. 1, Leipzig: Johann Wendler.

Hutcheson, F. (1986 [1725]): Über den Ursprung unserer Ideen von Schönheit und Tugend, Leidhold, W. (Hrsg.), Hamburg: Felix Meiner. 
Kennedy, G. (2011): The Hidden Adam Smith in his Alleged Theology, in: Journal of History of Economic Thought, Vol. 33/No. 3, 385-402.

Kennedy, G. (2013): Adam Smith on Religion, in: Berry, C. J./Paganelli, M. P. (Eds.): The Oxford Handbook of Adam Smith, Oxford: Oxford University Press, 465-484.

Leathers, C. G./Raines, P. J. (1992): Adam Smith on Competitive Religious Markets, in: History of Political Economy, Vol. 24/No. 2, 499-513.

Leathers, C. G./Raines, P. J. (2008): Adam Smith on Religion and Market Structure, in: History of Political Economy, Vol. 40/No. 2, 345-363.

Luterbacher-Maineri, C. (2008): Adam Smith - Theologische Grundannahmen. Eine textkritische Studie, Fribourg: Academic Press.

Mebta, P. B. (2006): Self-Interest and Other Interests, in: Haakonssen, K. (Ed.): The Cambridge Companion to Adam Smith, Cambridge: Cambridge University Press, 246-269.

Merikoski, I. A. (2002): The Challenge of Material Progress: The Scottish Enlightenment and Christian Stoicism, in: The Journal of the Historical Society, Vol. 2/No. 1, 55-76.

Nutzinger, H. G. (1991): Das System der natürlichen Freiheit bei Adam Smith und seine ethischen Grundlagen, in: Jahrbuch für Ökonomie und Gesellschaft, Bd. 9: Adam Smiths Beitrag zur Gesellschaftswissenschaft, Frankfurt a.M.: Campus, 79-100.

Oncken, A. (1898): Das Adam-Smith-Problem, in: Zeitschrift für Sozialwissenschaft, 1/1898, 25-33; 101-108; 276-287.

Oslington, P. (2011): Introduction: Theological Readings of Smith, in: ders. (Ed.): Adam Smith as Theologian, New York: Routledge, 1-16.

Oslington, P. (2012): God and the Market: Adam Smith's Invisible Hand, in: Journal of Business Ethics, Vol. 108/No. 4, 429-438.

Pabst, A. (2011): From Civil to Political Economy - Adam Smith's Theological Debt, in: Oslington, P. (Ed.): Adam Smith as Theologian, New York: Routledge, 106-124.

Paganelli, M. P. (2008): The Adam Smith Problem in Reverse: Self-Interest in the Wealth of Nations and the Theory of Moral Sentiments, in: History of Political Economy, Vol. 40/No. 2, 365-382.

Paganelli, M. P. (2010): The Moralizing Role of Distance in Adam Smith: The Theory of Moral Sentiments as Possible Praise of Commerce, in: History of Political Economy, Vol. 42/No. 3, 425-441.

Paganelli, M. P. (2011): The Theory of Moral Sentiments 1759 vs Theory of Moral Sentiments 1790: A Change of Mind or a Change in Constraint? in: Studi e Note di Economica, Vol. 16/No. 2, 123-132.

Paszkowski, W. (1890): Adam Smith als Moralphilosoph (Diss.), Halle an der Saale.

Patzen, M. (1991): Zur Diskussion des Adam-Smith-Problems - ein Überblick, in: Meyer-Faje, A./Ulrich, P. (Hrsg.): Der andere Adam Smith - Beiträge zur Neubestimmung von Ökonomie als Politischer Ökonomie, Bern: Haupt Verlag, 21-54.

Pesciarelli, E. (1999): Aspects of the Influence of Francis Hutcheson on Adam Smith, in: History of Political Economy, Vol. 31/No. 3, 525-545. 
Raphael, D. D. (1991): Adam Smith, Frankfurt a. M.: Campus.

Raphael, D. D. (2007): The Impartial Spectator. Adam Smith's Moral Philosophy, Oxford: Oxford University Press.

Raphael, D. D./Macfie, A. L. (1982): Introduction, in: Smith, A.: The Theory of Moral Sentiments, Indianapolis: Liberty Fund.

Rae, J. (1895): The Life of Adam Smith, London: McMillan \& Co.

Ross, I. S. (1998): Adam Smith - Leben und Werk, Düsseldorf: Verlag Wirtschaft und Finanzen.

Rothschild, E. (2002): Economic Sentiments. Adam Smith, Condorcet, and the Enlightenment, Cambridge: Harvard University Press.

Schumpeter, J. A. (2009): Geschichte der Ökonomischen Analyse, Bd. 1, Göttingen: Vandenhoeck \& Ruprecht.

Skarsynski, W. v. (1878): Adam Smith als Moralphilosoph und Schöpfer der Nationalökonomie, Berlin: Theobald Grieben.

Smith, A. (1982 [1795]): The History of Astronomy, in: Essays on Philosophical Subjects, Wightman, W. P. D./Bryce, J. C. (Eds.), Indianapolis: Liberty Fund.

Smith, A. (1985a [1759]): Theorie der ethischen Gefühle, Eckstein, W. (Hrsg.), Hamburg: Felix Meiner.

Smith, A. (1985b [1983]): Lectures on Rhetoric and Belles Letters, Bryce, J. C./Skinner, A. S. (Eds.), Indianapolis: Liberty Fund.

Smith, A. (1987 [1977]): Correspondence of Adam Smith, Mossner, E. C./Ross, I. S. (Eds.), Indianapolis: Liberty Fund.

Smith, A. (1990): Der Wohlstand der Nationen, Recktenwald, H. C. (Hrsg.), München: dtv.

Weber, M. (1988 [1906]): Die protestantischen Sekten und der Geist des Kapitalismus, in: Gesammelte Aufsätze zur Religionssoziologie, Bd. 1, Weber, M. (Hrsg.), Tübingen: J.C.B. Mohr.

Werhane, P. H. (2000): Business Ethics and the Origins of Contemporary Capitalism: Economics and Ethics in the Work of Adam Smith and Herbert Spencer, in: Journal of Business Ethics, Vol. 24/No. 3, 185-198.

West, E. G. (1976): Adam Smith - The Man and his Works, Indianapolis: Liberty Fund. 